\title{
LA COSTRUZIONE DELLO SPAZIO TURISTICO NELLA COSTA SMERALDA: NEOREALISMO O BANALIZZAZIONE DELL'ARCHI- TETTURA VERNACOLARE?
}

Alessandra CAPPAI

La Costa Smeralda nasce nei primi anni '60 nelle coste nordorientali della Sardegna, in un momento di grandi cambiamenti per il turismo. Mentre nel resto d'Europa il boom turistico del secondo dopoguerra significava la democratizzazione delle vacanze e la diffusione del turismo di massa, la Costa Smeralda nasce in antitesi alle altre destinazioni turistiche di massa sorte nelle coste spagnole, italiane e francesi dello stesso periodo. La condizione insulare e la natura incontaminata diventano simbolo dell'esclusività, che attraggono in Costa Smeralda clienti d'elite alla ricerca di un enclave paradisiaco di lusso nel centro del Mediterraneo. La rinterpretazione dell'architettura vernacolare e l'immanenza del paesaggio naturale saranno gli strumenti utilizzati per la costruzione dello spazio turistico.

Immaginario mediterraneo, vernacolare, regionalismo, pittoresco

\section{THE CONSTRUCTION OF TOURIST SPACE ON THE EMERALD COAST. NEOREALISM OR TRIVIALIZATION OF VERNACULAR ARCHITECTURE?}

Costa Smeralda was founded in the early 1960s in the north-eastern coast of Sardinia, at a time of great change for tourism. While for the rest of Europe the tourism boom after World War II meant the democratization of the holidays and the spread of mass tourism, Costa Smeralda was founded in opposition to the other mass tourism destinations fate in the Spanish, Italian and French coasts of the same period. The insular condition and the pristine nature of Costa Smeralda became a symbol of exclusivity, which attracted elite clients looking for a heavenly enclave of luxury tourism in the heart of the Mediterranean. The reinterpretation of vernacular architecture and the immanence of the natural landscape would have been the tools used for the construction of space tourism.

Mediterranean imagery, vernacular, regionalism, picturesque. 


\section{Inntroduzione}

La Costa Smeralda, ubicata nella regione storica della Gallura, non esisterà fino al 1962, data della creazione ufficiale della località turistica.

Nonostante il pioniere della Costa Smeralda sia di fatto Mr Duncan Miller, il suo ufficiale fondatore è il Principe Karim Aga Khan che, all'epoca 26enne, era già stato indicato come successore Immam degli ismaeliti (un'importante carica religiosa di una fazione di musulmani), e sarà per 30 anni Presidente del Consorzio Costa Smeralda.

L'Aga Khan decise di creare la Costa Smeralda alla luce di ciò che stava accadendo nella vicina Costa Azzurra, la cui esclusività andava poco a poco esaurendosi per via della sua posizione facilmente raggiungibile dagli altri paesi d'Europa, fatto che iniziava a snaturarne il carattere esclusivo. Il Principe riconobbe in questa situazione una possibilità straordinaria, e per questo volle creare un'altra area esclusiva come lo era stata la Costa Azzurra quindici anni prima, con la differenza che solo una clientela elitaria avrebbe potuto raggiungere un'isola. Agli albori di questo sviluppo, per attirare investitori e possibili acquirenti, l'Aga Khan aveva disposto un collegamento attraverso un volo charter, con il quale gli amici del Principe venivano accompagnati a visitare le terre dei Monti di Mola, che significa appunto Monti di Pietra, e che in futuro avrebbe preso il nome della Costa Smeralda.

Su proposta dell'Aga Khan si decise di formalizzare la nascita della Costa Smeralda attraverso la creazione di un Consorzio: invece di procedere secondo la pratica comune in cui ognuno degli investitori acquisiva e costruiva per conto proprio secondo esigenze e interessi personali, i soci del Consorzio si impegnavano a rispettare uno statuto ufficiale e delle norme comuni, a cui qualsiasi decisione avrebbe fatto capo. In questo modo si auspicava di creare qualcosa di speciale e di differente. L'atto costitutivo vero e proprio del Consorzio Costa Smeralda venne firmato il 14 marzo del 1962, alla presenza del notaio Altea e dei sei fondatori: Sua Altezza Aga Khan, Andrè Ardoin, Patrick Guinness, Bigio Felix, John Duncan Miller e René Podbielscki. Il consorzio si presenta come un'Associazione non lucrativa, "con lo scopo di programmare un equilibrato sviluppo urbanistico e residenziale e di dotarlo di opere di qualità necessarie per una migliore 
valorizzazione turistica" ${ }^{1}$; al principio era costituito solo dai sei fondatori ed era presieduto dall'Aga Khan.

Lo scopo del Consorzio era quello di riunire sotto un'unica gestione le ingenti inversioni previste per riuscire in un'operazione finanziaria a lungo termine. Ciò che avrebbe differenziato la Costa Smeralda da tutte le altre realtà turistiche sarde e molte altre europee è la forma consortile di gestione dei tremila ettari di territorio costiero: in questo modo si garantiva un livello di qualità più elevato, grazie al controllo dall'alto, effettuato su ogni elemento incluso nei limiti consortili. Per poter definire le norme edilizie, valutare i progetti e iniziare a realizzare i primi edifici della Costa Smeralda, venne costituito un Comitato di Architettura, formato da un equipe di architetti internazionali scelti dal Principe Aga Khan per avviare le prime strutture ricettive dei soci fondatori: tra essi Luigi Vietti rappresenta il più importante del Comitato.

\section{La costruzione dello spazio turistico: Porto Cervo.}

Al suo arrivo in Sardegna, Vietti viaggiò nell'Isola alla scoperta del territorio in compagnia del Principe Aga Khan, per raccogliere i caratteri degli insediamenti e dell'architettura tradizionale popolare che, mescolati, reinterpretati e rinominati, dovevano diventare gli elementi guida per la progettazione della Costa Smeralda.

L'obiettivo del comitato era, nei primi anni, quello di non intaccare la natura imponendole in modo violento l'edificato, cosa che peraltro avrebbe messo a rischio il carattere paradisiaco della destinazione turistica, dovuta proprio alla sua verginità. La necessità di non aggredire il paesaggio bilanciava la voglia degli architetti di dar libero sfogo alla fantasia. Come altri architetti che approdarono in Gallura, anche Vietti si trovava di fronte ad un territorio in cui tutto era permesso, e spettava alla sensibilità di ciascuno saper spendere quella libertà con cautela.

Conservando solo in parte la sua matrice razionalista, che lo inserì tra i protagonisti dell'architettura del regime fascista, cercò di riscoprire l'architettura povera, quella dei contadini e dei pescatori, riadattandola alle nuove esigenze.

Da qua l'associazione del lavoro in campo turistico di Vietti con le vicende del neorealismo, diffuse in Italia nel secondo dopoguerra a partire dal campo cinematografico fino all'architettura: la riscoperta delle tecniche costruttive, i saperi tradizionali, forme spontanee e legate alla vita contadina caratterizzano questa corrente, che già Vietti aveva sperimentato negli anni '30 in alcuni dei suoi lavori.

1 Presentazione del Consorzio nella pagina ufficiale www.consorziocostasmeralda.com 
In Costa Smeralda però l'intervento sarebbe stato totalmente artificiale, poichè quelle coste quasi non erano state intaccate dall'attività umana. Ormai è il genius loci a suggerire al progettista ciò che il territorio è in grado di ospitare e come, e solo l'analisi attenta del luogo permetterà realizzare un'architettura invisibile.

In questo articolo si cercherà di apportare alcune riflessioni teoriche sull'operato di Vietti, definito a monte "neorealista", partendo dalle sue dichiarazioni su quali siano gli elementi propri del territorio che l'hanno ispirato fino alle valutazioni del suo lavoro da parte della critica. Benchè l'obiettivo della ricerca sia lo studio del progetto del territorio turistico, per comprendere l'opera di Vietti nella Costa Smeralda è necessario partire dall'elemento architettonico per poi arrivare alla pianificazione dei nuclei. Il borgo di Porto Cervo parte infatti dalla costruzione dell'Hotel Cervo e le prime ville del Principe per poi articolarsi in pianta e in alzato con nuovi volumi, strade e piazze che si adattano alla conformazione del sito dando vita al "centro"; i piani di lottizzazione sono posteriori ai primi sviluppi, e nascono dall'esigenza di regolare l'edificato, la cui distribuzione era però già stata ideata.

Vietti dichiara che ciò che l'ispirò del territorio gallurese era la vegetazione aspra mediterranea, prevalentemente composta da ginepri, che al vederli da lontano sembravano formare una foresta, ma al passargli affianco un uomo con una mucca, essi perdevano la loro dimensione. Questo cambiamento di proporzioni nella macchia mediterranea lo indussero a voler fare delle "case che non si vedono". ${ }^{2}$

Laltezza delle costruzioni era un elemento fondamentale per integrare le costruzioni con il paesaggio circostante, avente come riferimento i ginepri alti massimo 2,50 m, e da rocce granitiche che creano movimento nel terreno. Le costruzioni potevano quindi raggiungere massimo due livelli, per cercare di nascondere le case nel paesaggio e non ostruire la visuale tra gli edifici. L'elemento visuale diviene fondamentale nella distribuzione dell'edificato in pianta e sezione.

La difficoltà di lavorare in terreni quasi sempre scoscesi doveva essere risolto con accorgimenti tali da non sommare tra di loro i piani. Se infatti su un piano inclinato si costruiscono delle case, anche solo di due piani, senza lasciare spazio verde tra una casa e l'altra, l'effetto ottico sarà comunque quello di un edificio multipiano; rispettando gli spazi, la prospettiva nasconde una casa nell'altra.

Anche l'uso di materiali originari del luogo che si integrino con la conformazione del territorio è importante per mantenere intatto il carattere locale: per gli elementi verticali si prediligono i blocchi monolitici tagliati di granito rispetto al cemento armato, tranne nelle strutture orizzontali, dove il cemento è alternato a travi in legno o archi di pietra. 


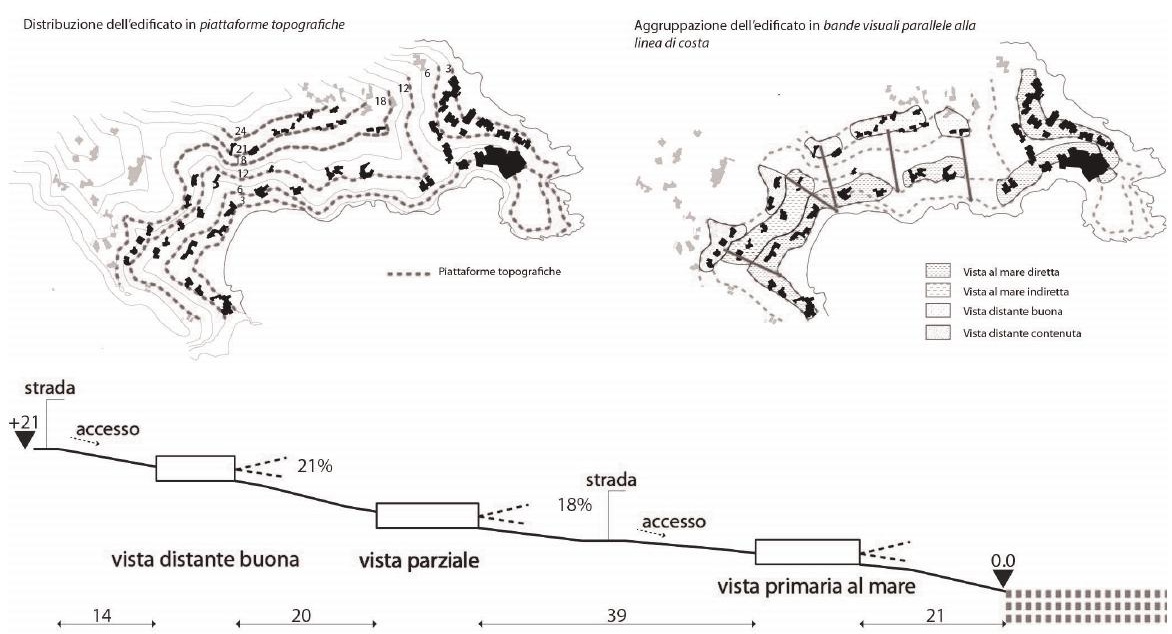

Fig.1: Planimetria e sezione trasversale dell'area a nord di Porto Cervo, che mostra l'inserimento visuale dell'edificato mediante l'adattamento topografico. Fonte: elaborazione dell'autore.

L'uso degli archi nelle architetture di Vietti era frequente già a partire dagli anni ' 30 nella Casa dei Pescatori alla Foce (1938); in quel caso tuttavia non vi era nessun tentativo di creare un borgo ex nihilo, anzi era la funzione che quel luogo aveva sempre avuto, la presenza umana che aveva caratterizzato quelle coste, a suggerirne l'uso. In quel caso l'architettura vernacolare esisteva davvero, ed era fortemente legata al mare e a quella popolare mediterraneità che invece era estranea alla Gallura. L'uso dell'arco verrà utilizzato soprattutto per distinguere il piano terra, generalmente un portico ad archi, dal corpo massiccio che su esso viene poggiato.

Anche i contrafforti, presenti in varie architetture smeraldine, sono un'eredità della Casa dei Pescatori alla Foce.

Nel caso di Porto Cervo, a differenza delle ville che cercano di nascondersi nel paesaggio e il Pitrizza che pretende mimetizzarsi in esso, le intenzioni di Vietti sono distinte: “(...) si deve sentire la cromatura delle abitazioni, si deve sentire la vita pulsante degli abitanti. Non ho fatto costruzioni alte, sempre al piano terreno, ma è prevalsa questa idea di fare delle cose cromate, a carattere visivo, anche bianche, il bianco è un colore no?, ma anche rosa, giallo, celeste, come si usa nel Mediterraneo".

Considerando che il principale punto di accesso è considerato il porto, Porto Cervo doveva essere facilmente riconoscibile dal mare, con l'aiuto dell'effetto cromatico. Gli edifici di Porto Cervo erano originariamente bianchi, e in una fase 

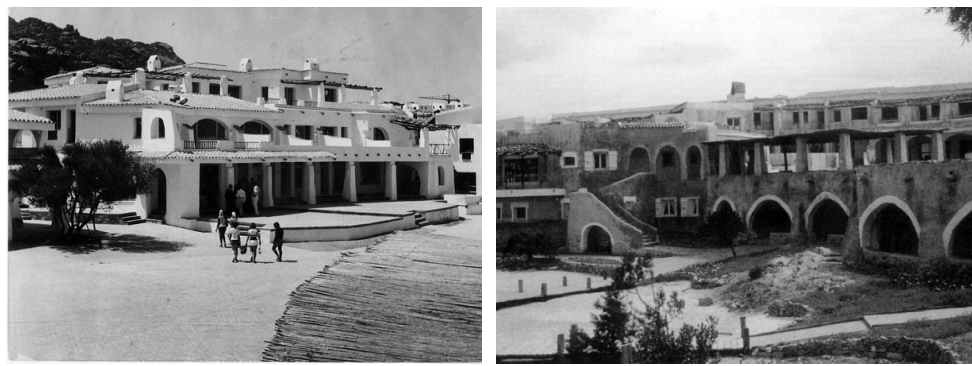

Fig.2: L'hotel Cervo e la piazza prima dell'invecchiamento. Fonte: Gerlat, 2003, La Costa Smeralda, il mito e il modello. Fig.3: L'hotel Cervo dopo l'applicazione del colore con effetto invecchiato. Fonte: Gerlat, 2003, La Costa Smeralda, il mito e il modello.

successiva erano pitturati con le diverse cromature e costretti ad un trattamento di finta invecchiatura che contribuiva a dare l'idea del "centro storico".

Sono inoltre presenti altri elementi ricorrenti nel paesaggio mediterraneo come i portici, le logge, i passaggi aerei sui vicoli, le scale esterne, i balconi e le verande, le cordonate ed i muri a scarpa, che sono da Vietti adattati alla sua architettura conferendogli un "tono pittoresco verso il quale induge senza pudori e pentimenti" " "Il risultato è un cocktail di esotismi ed elementi diffusi nella cultura mediterranea" (Barisone, Scelsi, 1999, p.36).

L' hotel Cala di Volpe di Couelle è considerata in realtà lo spunto per la creazione (Baumeister 1969) di questo stile esotico: come in una scenografia di un film, in esso sono presenti elementi di stile spagnolo, nordafricano, greco, che diviene la parola d'ordine per l'eclettismo delle successive costruzioni. Nasce uno stile "neosardo" totalmente artificiale.

Per riprodurre fedelmente l'idea di mediterraneità, Vietti adotta per il paesaggio turistico di Porto Cervo degli elementi tipicamente urbani facilmente ripetibili in altri luoghi: in questo caso si tratta di una o più piazze circondate da attività commerciali e ristorative, collegate tra loro da pittoreschi vicoli dove, attraverso la serie di porticati, scale e terrazze, lo spazio pubblico e quello privato, quest'ultimo costituito dagli alloggi dell'hotel Cervo e dai commerci, si integrano convincendo il turista di appartenere a questa scena di vita di "paese". Questo processo di adeguamento degli elementi urbani senza identità snatura tuttavia il carattere di spazio pubblico proprio della piazza, perchè sebbene aperto ai visitanti, Porto Cervo non è altro che un territorio privatizzato altamente controllato (Cappai, Alvarez, 2012).

La riproduzione di un borgo peschiero e dei suoi elementi più rappresentativi costituisce un'immagine turistica diffusa, in cui il porto diventa fulcro essenziale del villaggio. Non è difficile ritrovare il paesaggio di Porto Cervo 


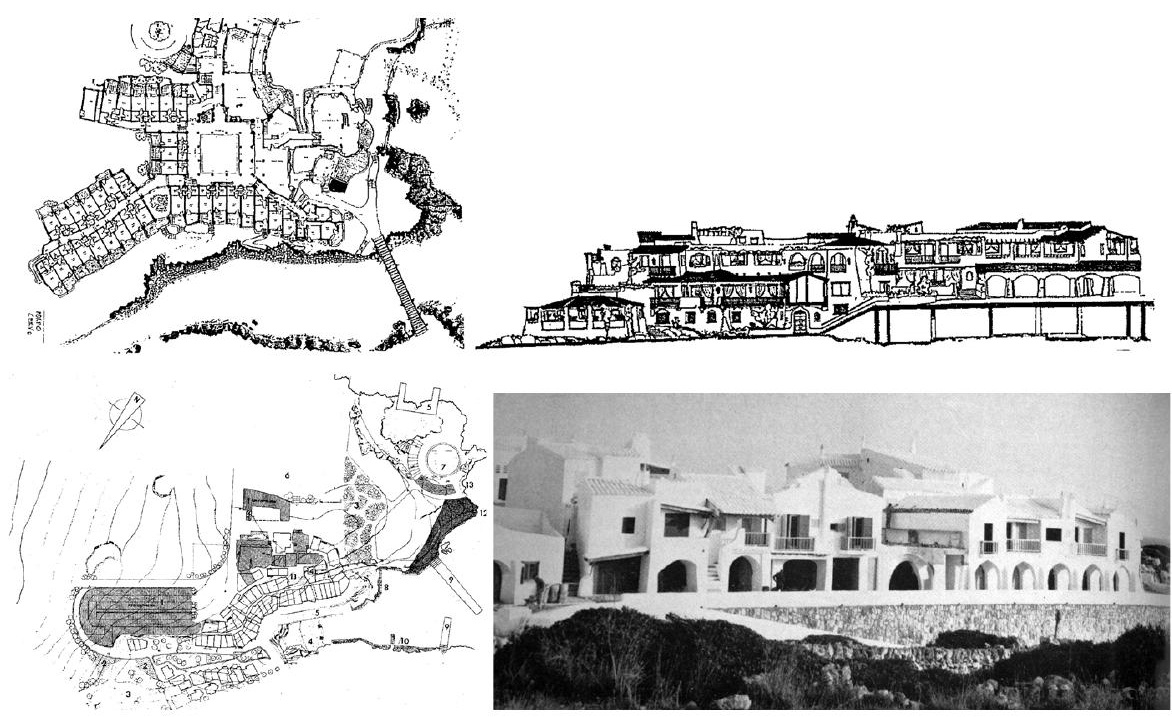

Fig.4- 5: Planimetria e prospetto del borgo di Porto Cervo. Fonte: Dell'Aira, 1997, Luigi Vietti: progetti e realizzazioni degli anni '30. Fig.6-7: Planimetria e fotografia del villaggio Binibeca a Minorca. Fonte: 1969, Architecture Française, 325.

ripetuto in altri luoghi turistici: è il caso di Binibeca nell'isola di Minorca dell'architetto Barba Corsini, in cui ritroviamo alcuni elementi identificativi quali il pontile, la piazza, la Chiesa, gli archi e i loggiati. Se non fosse per l'effetto invecchiato applicato agli edifici di Porto Cervo, sarebbe difficile distinguere le due località l'una dall'altra.

\section{Neorealismo o banalizzazione dell'architettura vernacolare?}

Difficile quindi affermare quale sia la vera base di riferimenti di Porto Cervo. L'acclamata riproduzione di un borgo peschiero, mediterraneo, dai colori pastello invecchiati, poco ha a che vedere con gli insediamenti preesistenti della Sardegna. Vietti stesso riconosce che, a differenza della Riviera, in Sardegna era difficile adattarsi ad un'architettura spontanea costiera, giacchè questa, nei pochi centri come La Maddalena, Arbatax o Torre Grande, non possedeva caratteristiche sufficientemente forti, e pertanto adattarsi al poco che esisteva avrebbe voluto dire agire semplicemente.

D'altra parte bisogna ammettere che il riferimento più vicino dell'architettura tipicamente gallurese, lo stazzo, non si prestava a subire un riadattamento stilistico: lo stazzo era legato ad un'economia pastorale molto povera, e la sua conformazione monocellulare o in alcuni casi bicellulare, non era adatta nè alla formazione di nuclei policellulari come residence o complessi alberghieri, e tantomeno alle ville di lusso. 

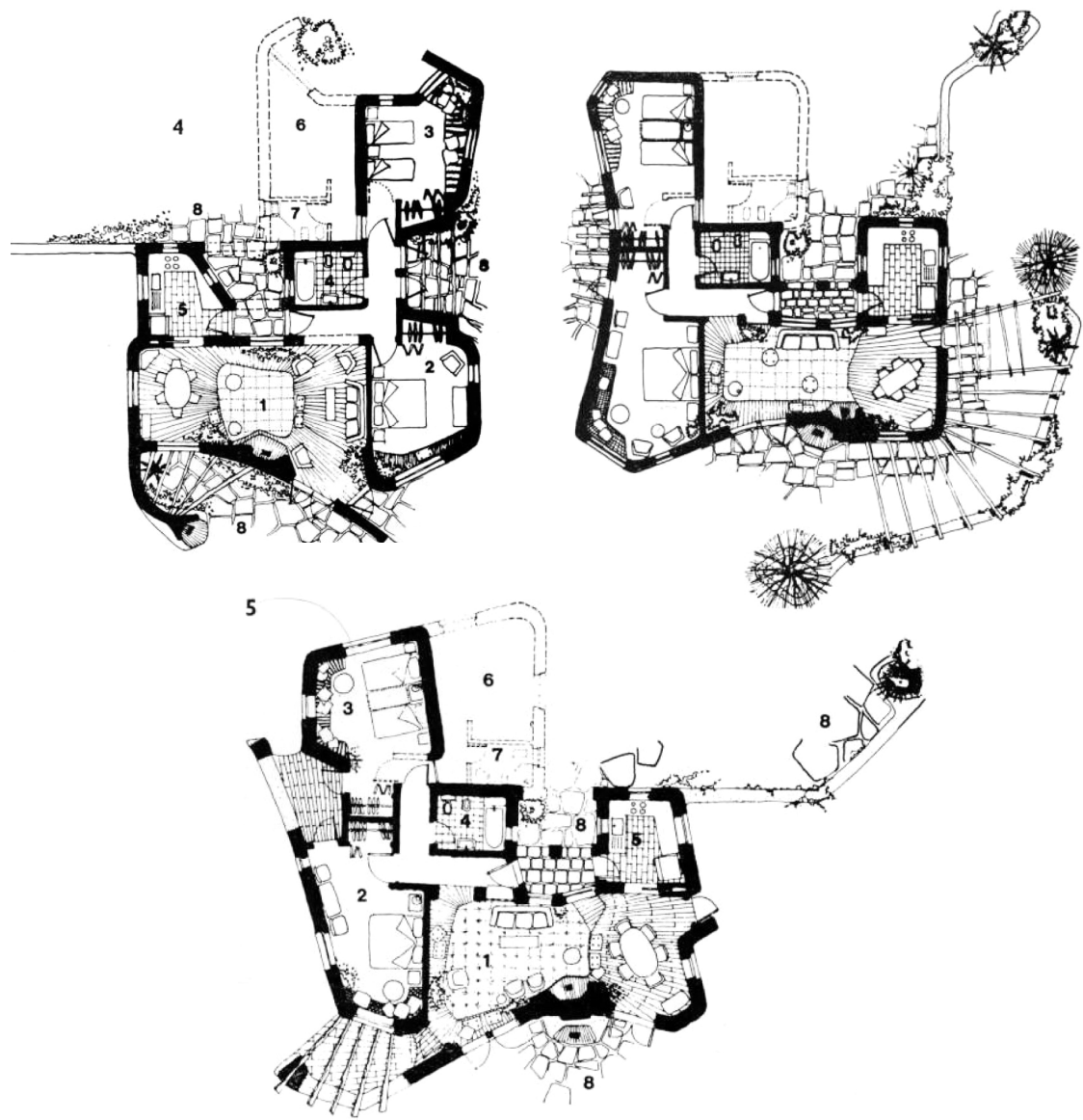

Fig.10: Planimetria di una villa dell'Hotel Cala di Volpe di Jacques Couelle. Fig.11: Planimetria di una villa dell'Hotel Cervo di Luigi Vietti. Fig.12: Planimetria di una villa di Michele Busiri Vici. Fonte: 1969, Architecture Française, 325 .

Non si legge nessun riferimento specifico, soprattutto alla Sardegna, ma piuttosto un insieme di elementi tratti dall'architettura popolare del mediterraneo, che simulano, soprattutto a Porto Cervo, l'“urbanità mediterranea", conseguita con una precisa architettura degli spazi, delle strade e delle piazze, e con la definizione architettonica (ma non semantica) dello spazio pubblico. Questi elementi, mescolati armoniosamente, devono inserirsi nella topografia del terreno senza causare un forte impatto; l'immanenza del paesaggio naturale rende più facile il lavoro dell'architetto. 


\section{Perchè allora mettere in relazione il neorealismo con Luigi Vietti?}

E' la Consorti a suggerire la relazione tra il caso della Costa Smeralda e il movimento neorealista, individuando un filo comune tra le opere neorealiste e certe operazioni turistiche, tra cui il borgo di Porto Cervo: "Forme elementari, materiali comuni-intonaco, tufo, pietra locale, legno-assenza totale di aggettivazione, traducono simbolicamente in architettura la dignitosa povertà di una società frugale e antica e annunciano la speranza di un progresso capace di non cancellare la tradizione" 5 .

Nonostante non vi sia, nel caso di Porto Cervo e le architetture di Vietti nella Costa Smeralda un riferimento ad un modello preciso, si deve riconoscere che con l'adozione di certi elementi non si discosta totalmente dalla tradizione, e forse si potrebbe pensare che si tratti di un'evoluzione del modello tradizionale che l'utente stesso avrebbe adottato per modificare il proprio habitat tradizionale (Trillo 2003). Considerando l'"effetto Costa Smeralda" in tutta la Sardegna, non solo nelle coste e non solo nell'architettura turistica, forse bisogna ammettere che così è stato, anche se non con esiti del tutto positivi.

Tuttavia nel caso dei villaggi turistici e case per vacanze questi elementi vengono esasperati, sino a comporre un "esperanto vernacolare" (Consorti 1997) riproducibile in tutte le coste. Porto Cervo diventa quindi un caso di nuova fondazione di un territorio con l'illusione di riprodurre un'autentica mediterraneità, sconcertando la critica per questa evidente alterazione di significato.

Se da una parte le scelte progettuali deformano definitivamente le tipologie insediative locali in apparenza condividendone alcune manifestazioni superficiali, le politiche di marketing cercano di esaltare quest'aspetto vernacolo e d'isolamento insulare che attrae la ricca committenza (Carta 2007). Si forma così un modello turistico che si estende in tutte le coste alla base della malinterpretazione dell'architettura tradizionale locale.

Il paesaggio turistico costruito si sovrappone al patrimonio architettonico esistente, producendo un fenomeno di banalizzazione del paesaggio locale.

Definendo l'opera di Vietti neorealista tuttavia, è necessario fare riferimento non solo al tentativo di recupero dell'architettura locale spontanea, obiettivamente poco riuscito se non fosse, come abbiamo visto, per l'idoneo utilizzo di certe caratteristiche puntuali quali gli archi o i materiali naturali, ma piuttosto ai suggerimenti del territorio che Vietti sembra capace di cogliere.

Dall'analisi dei piani emerge che il suo approccio sistematico e dettagliato alla conoscenza della natura del terreno, la sua morfologia, i venti, sono i segni del suo "neorealismo". 
Non solo l'assetto ambientale, ma anche le tracce preesistenti dell'uomo costituiscono la base per lo sviluppo di Porto Cervo: i sentieri sono lo spunto per il tracciato delle strade, che vengono disegnate quasi manualmente sul territorio, e i muri a secco diventano i limiti delle lottizzazioni o dei lotti stessi.

\section{Conclusioni}

Il neorealismo di Vietti è da intendersi dunque come l'insieme della raccolta degli elementi naturali e antropici del territorio preesistente, per la fondazione di un territorio che invada meno possibile il paesaggio originario, attraverso l'adozione di uno stile mediterraneo che sebbene si discosti da quello propriamente locale, riesce a Porto Cervo nella creazione dell'urbanità (anche se temporanea e elitaria).

I risultati raggiunti da Vietti alla ricerca dell'architettura spontanea in Sardegna, sono molto lontani dal resto degli interventi neorealisti della penisola, di cui un esempio chiave è il quartiere Tiburtino a Roma di Quaroni, in cui residenze convenzionate sono promosse per il miglioramento della vita urbana e dell'habitat sociale. Nella Costa Smeralda il paradosso è quello di una clientela ricca che vuole abitare in ville di stile "povero".

Sebbene si riconosca che l'intento di Vietti di riprodurre un'architettura vernacolare sia un'illusione, una sorta di confusione della clientela che è convinta di trovarsi di fronte ad un paese vissuto, non si è d'accordo con la critica comune che afferma che non vi fu nessun confronto diretto con gli orientamenti dei percorsi, le orografie e le esposizioni, ovvero con il territorio e la natura circostante. Pur arrivando a discreti risultati formali, soprattutto nella composizione finale di Porto Cervo, vi fu un'attenta analisi del territorio, inteso come elementi naturali, conformazione del terreno, vegetazione e scorci paesaggistici, e non un "rimando semplicistico al contesto". 


\section{Bibliografía}

AA.VV. (1973). "I neo-paesi, in Abitare". oํ 119 (pp. 94-109).

BARISIONEM S., SCELSI, W. (1999). "Luigi Vietti: Architetture liguri". Genova: Erga Edizioni.

BIANCHI, G., GIARDIN, G. (1999). "Case di Sardegna”. Milano: Ed. Archideos.

CAPPAI, A., ALVAREZ I. (2012). "Le seconde case in Sardegna: un potenziale modello urbano e turistico per il futuro del litorale". In L'architetto italiano, 48 (20-23). Roma: Mancosu Editore

CARTA, M. (2007). "La sottile linea blu. Insediamento costiero e progetto di territorio. II caso gallurese". Cagliari: CUEC.

CONSORTI, C. (1997). "Roma, Napoli, la Sicilia". In F. DAL CO, a cura di, Storia dell'architettura italiana. II Secondo Novecento, Vol.II. Milano: Electa.

DELL'AIRA, P.V. (1997). "Luigi Vietti. Progetti e realizzazioni degli anni '30". Firenze: Alinea Editrice.

GERLAT, S. (2003). "La Costa Smeralda. II mito e il modello". Sassari: Carlo Delfino Editore.

TRILLO, C. (2003). "Territori del Turismo, tra utopia e atopia". Firenze: Alinea Editrice.

VON SCHWARZWE, D. (1969). Neosardischer Stil = Florasarda + Stradasarda + Alisarda+ Biancasarda + Cerasarda+ Marinasarda= Costa Smeralda. In Baumeister, 8 (1003-1009). 